\title{
EXPERIENCIA MORAL Y ACCIÓN COMUNICATIVA ${ }^{1}$
}

\author{
Humberto Giannini \\ Con la colaboración de Juan José Fuentes, \\ Eva Hamamé, Isabel Álvarez y Nicole Gardella \\ Universidad de Chile \\ gianninluz@mi-mail.cl
}

\begin{abstract}
Resumen
La comunicación está referida a la categoría de la acción, pero no dirigida inmediatamente al mundo, sino a promover algún tipo de respuesta en otro sujeto. Y es este campo limitado -dominio de la interacción humana- el objeto propio de la ética.
\end{abstract}

PALABRAS ClaVE: teleología, universalidad, acción comunicativa, referente, sujeto de la acción, experiencia moral, interacción, intencionalidad.

\section{Abstract}

Communication refers to the category of action, but it is not directed to the world immediately. Instead, it is oriented to elicit a certain type of answer from somebody else. This constrained field-domain of the human interaction-is the particular subject of Ethics.

KEYWORDS: Teleology, universality, communicative action, reference, agent, moral experience, interaction, intentionality.

\section{El problema de la ética}

$\overline{R A}$

En una mirada histórica, la ética puede presentarse incluso hoy como una reflexión acerca del bien humano. Se comprende que esta presentación 'marcaría el paso' en el mismo punto si no se mostraran las diversas significaciones, incluso incomposibles, que ha venido asumiendo este término de 'bien'.

Fue Aristóteles quien edificó el saber ético sobre el presupuesto de que todo ente natural tiende por su propia constitución interna hacia un fin, que es, al mismo

Este texto es resultado de la investigación desarrollada en el proyecto FONDECYT 1070936, actualmente en curso, "Acción comunicativa y experiencia moral. Esbozo para una ética del conflicto". 
tiempo, el bien del individuo y de la especie a la que ese individuo pertenece. "Por eso, con justa razón se ha declarado que el bien es aquello a lo que todas las cosas tienden" (Aristóteles, Etica Nicomachea, 1094a 4). Y tienden a él por naturaleza.

En primer término, es el bien -lo amable en sí- la causa de esa suerte de anhelo cósmico -"el Amor-dirá Dante- que mueve al Sol y al firmamento" (Divina Commedia, Parad. XXXIII, 145)- el cual, comunicándose a cada ente del Universo, aguijonea su naturaleza para que todos y cada uno participen, en la medida de su fuerza, de la vida eterna del ser.

Es en virtud de este impulso a la eternidad que el filósofo explica, luego, la conducta, irracional pero certera, de los animales: “...Y es que para todos los vivientes que son perfectos -es decir, los que no son ni incompletos ni tienen generación espontánea- la más natural de las obras consiste en generar otro viviente semejante a sí -si se trata de un animal, otro animal, y si se trata de una planta, otra planta-con el fin de participar de lo eterno y lo divino en la medida en que les es posible; y con tal fin realizan cuantas acciones realizan naturalmente" (Arist., Dell'Anima, 415a 25).

Ahora, a propósito del ser a cuya naturaleza pertenece el actuar por elección deliberada - proairesis- es conocido el argumento aristotélico que dice que si este no tuviera a la vista un fin final, un bien último al que supeditara todos los otros, entonces, los fines que vienen por añadidura quedarían desamarrados y se derrumbarían como carentes de valor y de sentido: "Porque no elegimos cada cosa en razón de otra, pues de esta manera se procedería al infinito, de modo que el deseo humano sería vacío e inútil" (Arist., Etica Nicomachea, 1094a 20).

Así, el hombre se encamina hacia el bien último que le es connatural-hacia lo Inteligible- solo cuando elige las acciones según los dictados de su naturaleza específica: la inteligencia, la razón.

En definitiva: es el fin final lo que mueve tanto la conducta irracional de los animales como la acción y el anhelo racionales del individuo humano: "Porque, en la medida de lo posible, nosotros debemos volvernos inmortales" (Arist., Etica Nicomachea, 1177b 30).

Hay, pues, en la historia del pensamiento occidental una ética que pretendió demostrar la existencia necesaria de ese bien Universal 'hacia el que tienden todas las cosas', y hacia cuyo goce se encaminan las acciones del hombre sabio. Se trata de una ética teológica y, a la vez, teleológica, ligada fuertemente a Aristóteles y sus discípulos medievales ${ }^{2}$.

2 También se puede hablar de una Ética teleológica ligada al conocimiento 'verdadero' de Dios, del Logos, de la Naturaleza. Es el caso del pensamiento estoico, sobre todo, en su etapa romana ('nuevo estoicismo'), y que repercutió fuertemente en los primeros tiempos del Evo moderno. ¿No existe incluso en 'el primer Heidegger' esta dimensión teleológica en su idea de 'proyecto auténtico'? 
No obstante, algo sustancial cambia al interior de esta misma concepción tradicional con el advenimiento de la teología judeocristiana y su eje inconmovible de un Dios creador del Universo. En la nueva visión creacionista no bastará sostener que las cosas son buenas porque 'por naturaleza' tienden al bien. Para el medioevo, las cosas tienden al bien porque ab initio son buenas: porque son criaturas de Dios (Esse creatum bonum est qua creatum). Desde estas premisas resultaba impensable una ética ajena al discurso teológico, así como, desde este momento, también resultará inevitable hacerse la pregunta dramática por el sentido del mal en el mundo.

Con el oscurecimiento de la explicación teleológica (de la causa final), en el Evo moderno, la ética buscará adecuarse al nuevo concepto de naturaleza y, entonces, al nuevo concepto de naturaleza humana, en el que las ideas de fuerza interna y de causalidad funcional vienen a desempeñar un rol protagónico como condiciones inmanentes de la acción (impulsos, egoísmo, autoafirmación, voluntad de poder, espíritu gregario, libido, etc.).

El problema es que la ética, como saber determinado, no se limita a ser una mera descripción psico-biológica de la vida humana tal como esta es 'naturalmente'; por lo que no ha podido renunciar del todo a su antigua pretensión de mostrar cómo ese ser racional, social, lúdico, etc., que es cada uno de nosotros, debe emplear, dosificar y jerarquizar las fuerzas que posee $a$ fin de realizar el ser que debiera ser. Y cuál es la jerarquización valórica de sus facultades y energías, y mirando a cuáles principios, a fin de lograr el desarrollo armonioso y próspero de la especie humana.

Así, incluso desde este nuevo punto de partida mundano, inmanente, parece irrenunciable para la reflexión ética la idea de finalidad (proponernos prácticamente algo a fin de..., v. gr., proponernos ‘el más propio ser sí mismo’) Y precisamente aquí reaparece el conflicto original del pensamiento humano en su búsqueda del bien.

Puede plantearse de esta manera: en la ética, como disciplina práctica que es, no se trata solo de pensar coherentemente la validez universal del bien en el que creemos $^{3}$; su exigencia es la de realizar esta universalidad en cada una de nuestras acciones: "Puesto que el presente estudio no tiene por fin un conocimiento puro, como los otros (en efecto, no emprendemos esta búsqueda para conocer qué es la virtud, sino para volvernos buenos), es necesario examinar todo lo que concierne a las acciones para saber cómo es preciso realizarlas" (Arist., Etica Nicomachea, 1103b 27).

Quien no realiza el bien que predica, no cree en él o al menos no cree con una fuerza capaz de hacer creíble su pensamiento.

La pregunta es, pues, la siguiente:

¿Cómo es posible actuar éticamente, es decir, realizar en nosotros mismos el principio práctico que teóricamente sostenemos como universal? 
Se comprende que esto no es posible imponiéndonos un fin final, al que toda acción humana debiera dirigirse. Imposible, porque no hay ninguna razón para imponer a otros como universal el bien concreto que hemos elegido; simplemente porque no existe un objeto del mundo capaz de imponerse por sí, universalmente, como fin final. Y si lo hubiera, entonces, lógicamente, ya no sería un objeto de elección, sino un principio incontrovertible.

En otras palabras: el Evo moderno descubrió que la tarea de la ética no es buscar y proponer un objeto (un referente óntico) como bien universal, porque tal universalidad tendría solo una validez empírica que podría desecharse en cualquier momento histórico.

Concretamente, fue Kant quien descubrió que la ética no depende en su constitución de los referentes objetivos que un determinado grupo pueda tener como 'su Bien' (o como 'su' Dios), sino de que en todo momento el sujeto ético actúe ante otro sujeto de acuerdo con un imperativo impuesto a su acción: tratar a ese sujeto con el que nuestra acción se relaciona como un fin en sí mismo; jamás como un medio. La ética se ejerce, así, actuando, pero no directamente en el mundo, en busca de algo en el mundo, sino ante otro.

En resumen, dado el oscurecimiento de la metafísica, al menos el de la metafísica de 'la Causa final', la ética del anhelo tiende a transformarse en la búsqueda de una exigencia universal por la cual los individuos, al buscar cada uno su propio bien personal, no entorpecen con su pretensión el derecho ajeno a buscar el propio; y este respeto hace de la justicia y de la equidad la norma y la ley por la que deberán regirse todos los individuos de una sociedad bien constituida.

En otras palabras: en su versión moderna la ética no tiene que ver con la felicidad ni con algún fin puesto en el mundo o más allá del mundo, sino con el deber de reconocimiento al otro, como condición incondicionada de la acción (Kant).

Con todo, esta nueva perspectiva, al pretender cerrarlo, reabre un problema que la tradición metafísica creía tener resuelto. Un problema tensional originario, diríamos. Y es el siguiente: que haciendo todo el bien que según las prescripciones éticas debería a mi prójimo; suponiendo además la exacta retribución por parte de este, incluso así, es del todo posible que el bien que sigo anhelando -y que, por lo demás, nadie me debe ${ }^{4}$ esté en otra parte: por ejemplo, en la contemplación de las cosas superiores que según Aristóteles, nos acerca a los dioses (Arist., Etica, op. cit, 1082b 3), o en la exaltación de la belleza y del arte, o en el proyecto existencial de 'ser sí-mismo' en el modo más propio y auténtico (Cfr., Heidegger, Ser y Tiempo, Secc. II, cap. II) o, por qué no, en la aspiración infinitamente más modesta de permanecer unido al ser que amo. Y que este bien privilegiado incluso esté en conflicto con el deber abstracto de reconocimiento. 
En resumen: la búsqueda del bien y del sentido -ética de la sabiduría ${ }^{5}$ - no se compagina ni en su objeto ni en la disposición anímica con la que el sujeto lo enfrenta, con el imperativo ético del reconocimiento.

Los 'otros' sub specie son 'mis semejantes' (seres racionales, como yo). Pero, in praesentia, son aquellos respecto de los cuales estoy permanentemente expuesto, en los efectos, cercanos o próximos, de sus iniciativas. Es decir, la humanidad abstracta de los otros está aquí en la estrecha vecindad que constituye mi prójimo.

Hay razones fundadas para temer al prójimo, para desconfiar y cuidarse de él. Ninguna 'razón racional' (ni teológica, ni religiosa) para amarlo; y, a veces, 'el deber', se dice, de enfrentarlo: como enemigo, como competidor o como extracomunitario.

Mirada ahora desde la alta cúspide del deber-incluso del imperativo kantianola ética sigue apareciendo, entonces, más bien como el límite, como la condición estratégica, pactada, a partir de la cual el individuo puede retirarse en la intimidad domiciliaria, sin 'hacer mal a nadie', con la satisfacción interior del deber cumplido ${ }^{6}$.

Llamaremos 'concepción subjetivista' a la concepción tradicional. Incluso si para ella el bien trasciende toda subjetividad, como es el caso del Summum ens, del Summum bonum. Es subjetivista en cuanto cada sujeto se encuentra en una relación personal, directa y aislada con ese bien.

La segunda concepción, la del deber-ser, es subjetivista por otra razón: por imponer el bien como una relación del sujeto empírico a un principio ineludible de coacción interna. En el fondo: a una relación consigo mismo.

¿Cómo salir de un subjetivismo que, en ambos casos, se revela como extraño -o lejano- a la trascendencia que implica el bien o el Otro en cuanto prójimo?

La ética no es un saber estrictamente teórico, sino un saber de sabiduría (phrónesis). Esta condición se revela al menos en dos aspectos fundamentales: a) no tener referencia directa a los 'hechos del mundo'7; b) no recurrir a principios universales, apriorísticos, que no puedan encontrarse o descubrirse a partir de la experiencia que es propia de su campo (la experiencia del otro).

Este último punto se podría ilustrar así: es posible que un sujeto tenga muy confusa esa máxima que serviría de guía y de norma inapelable en relación con todas

$5 \quad$ La phrónesis de Aristóteles.

6 "La exigencia kantiana de encontrar, analógicamente, una legalidad en el reino de lo moral, reino de lo suprasensible, en vista de la legalidad siempre hallable en lo fenoménico, implicaría, entonces, un simple deseo de la razón, según el cual la potencia del sujeto que 'prescribe' sus leyes a la naturaleza podría ser efectiva también respecto de sí mismo. Así, el sujeto moral de Kant sería autónomo solo en cuanto legisla sobre sí mismo y puede hacerlo, siendo por ello sujeto y legislador. Pero tal atribución es desmentida inexorablemente por el carácter trascendente de la acción (Juan José Fuentes 2007)".

7 En el sentido de Wittgenstein: "El mundo se divide en hechos", Tractatus logico-philosophicus, 1. 2 . 
sus acciones, o que no se haya planteado seriamente en qué consiste, en términos universales, absolutos, ese reconocimiento incondicionado que debería a la humanidad del hombre en cuanto tal.

Sin embargo, no por esta carencia de un conocimiento aislado, abstracto, de los principios universales de la acción, podríamos afirmar que las acciones más propiamente humanas que cumple tal sujeto en relación con su mundo ambiente y con su prójimo, carezcan de una exigencia ética en el sentido más propio. Por ejemplo, cuando 'da su palabra', cuando 'pide', cuando 'ofrece', o simplemente cuando 'afirma algo' (testimonialmente). No podríamos negar que en el actuar mismo ante el otro, en la significación puntual de lo que hace, está presente una experiencia de la eticidad de su relación. Y no por el imperativo de una razón universalmente aplicada desde lo alto, sino de una experiencia ética primaria, básica, que surge allí mismo a raíz del significado de cada encuentro.

\section{La experiencia moral}

Un principio universal es tal en cuanto está presente en cada cosa singular, constituyéndola en esa singularidad. Nuestro trabajo quiere mostrar que la comunicación, en cada uno de sus infinitos modos y particularidades, es experiencia del otro $-s u$ reconocimiento en cuanto sujeto. $\mathrm{Y}$ a este reconocimiento empírico, carnal, es a lo que llamamos experiencia moral: inseparable de la experiencia que tengo de aquel con el que me comunico.

Tal experiencia no se gana como ese continuo experimentar que al cabo de tantas repeticiones se consolida como experiencia de vida, como hábito. Se conquista en la percepción inmediata que se tiene del prójimo. Percibir al otro 'ante mí' es ya percibir que el otro me percibe. El conocimiento del otro se inaugura, nace como reconocimiento. Se podría también decir que el conocimiento del mundo va construyéndose a partir del reconocimiento de los otros ${ }^{8}$.

Primera observación: el acto noético de mi propia percepción no es un hecho que se me dé inmediatamente como acto de mi conciencia. Al mostrarse el objeto, mi percepción -en el ver, por ejemplo- queda como el fotógrafo detrás de la operación. Solo un acto reflexivo posterior la hace avanzar hacia el primer plano.

Ahora, el acto de ver y la acción de mirar son realidades inseparables pero distintas. Yo no veo el ver (el acto intencional) del otro, pero 'veo' su mirada, la que de alguna manera me lleva a su modo de verme. Nos encontramos en la mirada para vernos. Esto es propiamente 'ser ante otro'. Nos encontramos con el otro en el espacio mundano de la interacción. 
Segunda observación: la ética, como teoría, es un saber distinto de otros saberes, justamente porque la experiencia moral es experiencia de aquel ente que comunica su subjetividad con la mía a través del mundo -incluyendo nuestros cuerpos significantes. Por eso, comunicarse es un esfuerzo permanente de aclaración.

Aún no se ha dicho lo esencial sobre este punto: en la comunicación de los sujetos hay, además, -pero con un 'además' que es estructuralmente insuprimiblealgo de ser que se trae a la presencia común. Una mirada vacía-que no pretendiera mostrar nada- es todavía comunicación: justamente, una mirada que pretende no mostrar nada.

Tercera observación: esta distinción estructural nos permite separar todo saber sobre referentes (obiecta) del saber propio de la ética. La ética estudia acciones comunicativas en acto tales como afirmar, negar, proponer, prometer, vaticinar..., acciones que están explícitas o implícitas en todo lo que se comunica ${ }^{9}$. En otras palabras, la ética tiene como campo de su estudio a los agentes permanentes del 'traspaso de mundo’ que ocurre en cada acción comunicativa. Volveremos sobre esto.

\section{La comunicación}

La comunicación, en tanto acción, es siempre 'la experiencia que se tiene de otro sujeto'. Se trata de una experiencia sui géneris, entre otras características, porque no se trata propiamente de una experiencia contemplativa, y este rasgo obliga a revisar, se comprende, las categorías habituales con las que se describe una realidad 'simplemente ante los ojos'. Se trata de una experiencia de la acción, sufrida y ejercitada a la vez, en cuanto el otro es co-actuante: aquel que está re-accionando y actuando ante mi propia acción. Por el momento, nos referimos también a acciones comunicativas no necesariamente verbales; a aquellas que se dan en la temperatura afectiva de los primeros gestos no dirigidos a referentes del mundo (una sonrisa, por ejemplo). Es en su ejercicio -o goce-, que esta experiencia se va espacializando, y creando con otros sujetos, un campo común de referencias: deíctico, primero, nominal, después, finalmente, dialógico.

Cuando se habla en general de comunicación, centramos nuestra atención en el referente (el 'objeto' que 'traemos' a la comunicación). Y así, marginamos la acción misma. En primer lugar, habría que devolver el término 'comunicación' a su real categoría ontológico-gramatical, a la categoría de acción. Comunicar es una acción. La acción del sujeto que, hic et nunc, la está realizando. El sujeto que comunica es siempre un yo actual, presente, pues en caso contrario se habría cortado la comunicación y nadie lo sabría. Para poner un ejemplo: si digo 'Juan me comunicó su renuncia', el último sujeto que está comunicando ese hecho soy yo, cuando digo, informando a 
alguien: 'Juan me comunicó su renuncia'. Y se comprende que toda comunicación tiene un sujeto actual que hace 'circular' algo del mundo, al descubrir o repetir o enseñar a otros el primer descubrimiento.

La acción comunicativa en su estructura está constituida por la acción misma de comunicarse un sujeto con otro sujeto, y por aquello que hemos llamado 'referente': aquello que se comunica, que se hace común (L comunica $q$ a L'). A través de la comunicación, el referente es aquello que se pone ante los ojos de L', eso mismo, supuestamente $^{10}$, que L quiere mostrar.

\section{La relación entre acción y referente}

Hacer aparecer algo para un otro es mostrarlo, 'ponerlo ante los ojos'. La comunicación tiene una función apofántica, pero no en el sentido de denotación ${ }^{11}$ que le dio el positivismo lógico (e incluso Aristóteles, en su Peri Hermeneias). La apófansis es la acción de revelar, de mostrar algo, mostrando-se.

¿Qué es lo que se muestra? Una relación objetiva tenida por verdadera, una situación, algo que pasa, un estado de ánimo, una disposición hacia el otro, el ejercicio actual de una prerrogativa del que habla, y que inviste al que escucha de cierto ser o cualidad de ser (Dios, por ejemplo, en una 'acción comunicativa'-Fiat!-, y solo en virtud de una acción comunicativa, otorgó el ser al Universo).

En primer término, es constitutivo de la acción de comunicar, hacerlo ante y para otro(s) sujeto(s) presente(s). En la proposición 'Yo comuniqué a Juan mi decisión de renunciar' no me estoy dirigiendo a Juan sino, por ejemplo, al sujeto carnal José, a quien ahora comunico o simplemente cuento aquella decisión.

Así, si bien es cierto que el referente puede pertenecer a cualquier tiempo real o posible, la acción de comunicar es como el instante aristotélico: es siempre presente; es en el 'ahora (nyn)' del yo histórico que, comunicando, va articulando y traspasando el mundo de las referencias. $Y$ en este sentido puede afirmarse que el mundo y el tiempo mundanos 'corren', 'circulan' a través de la comunicación de un presente que se mueve, de un presente que no está dirigido al mundo sino que es presente (don) 'para otro sujeto'.

La relación esencial es, pues, la co-presencia actual de los sujetos de la interacción comunicativa. Llamamos a esta interacción 'ser ante el otro'. Se trata de un modo recíproco de ser, de la proximidad del prójimo. Esto es, la máxima cercanía en que pueden encontrarse dos seres humanos.

10 Si es o no lo mismo, solo lo podrá revelar la historia de aquella concreta comunicación.

11 Esto es, ligada a la verdad o falsedad. 
Hay que destacar este hecho: en el vis-à-vis, el otro se vuelve una realidad sin perspectivas, sin escorzos: inmediatamente frente a mí, mirando mi propia mirada, buscándome en ella. Y a tal punto que sostener la mirada mutua de uno en el otro, más allá de cierto tiempo, se vuelve insoportable ${ }^{12} \mathrm{y}$, entonces, las miradas tienden a dispersarse hacia algún referente a la mano.

\section{El referente}

Cuando uno se pregunta por la relación entre los sujetos y el referente que se comunican, se está haciendo, en el fondo, una pregunta muy difícil de responder, a causa de la complejidad del asunto. Pero hay una respuesta que cubre, en casi todos los casos, las otras respuestas posibles: nos estamos preguntando qué acción intenta hacer un sujeto a fin de promover una repuesta en el otro. Algunas de las posibilidades son:

a) Un sujeto L intenta poner algo ante los ojos de otro(s) (L'): indicarle algo que pasa, un fenómeno, recordarle algo, darle una noticia, trasmitirle un conocimiento, en fin, algo digno de mención y de lo cual el sujeto L' no parece haberse percatado.

En todos estos modos de ser, cotidianos o científicos, la importancia del referente, la supuesta verdad de lo que allí se dice, vuelve, en la mayoría de los casos, 'comprensible de suyo', desechable entonces, el explicitar la acción misma de que 'eso' es algo que se está mostrando, recordando, enseñando. El valor independiente de la supuesta verdad - $\mathrm{S}$ es $\mathrm{P}$ - hace innecesaria la 'firma' de quien ahora lo dice o lo repite. Se procede como si las proposiciones fueran estructuras autosuficientes; como si no fueran, como lo son, un momento único de la comunicación humana, un momento en el que la atención de los hablantes converge hacia tal referente, entre los infinitamente posibles. En resumen: algo que es, por tanto, verdadero, independiente de que un sujeto lo denote o no.

Hay grados de intensidad en este dirigirse: desde la enunciación, que simplemente dice lo que es, hasta acciones como anunciar, jurar (juro que fue así), declarar, testimoniar, sostener, modos con diversa temperatura intencional (o fuerza ilocucionaria) por los que un sujeto da fe de algo. Otras: como el simple anunciar o la acción de vaticinar, profetizar, son modos de hacer común un ser, un hecho por-venir, supuestamente verdadero, también independientemente de que se lo mencione o no. Cuando la lógica se refiere al lenguaje denotativo, se refiere a tipos de acciones comunicativas ( $\mathrm{S}$ es $\mathrm{P}$ ), cuyo referente es susceptible de ser verdadero o falso. Pero, como hemos dicho, no son las únicas ni las más frecuentes en el discurso común. 
b) Por la acción comunicativa, L quiere garantizar a L' que en el futuro cumplirá una acción determinada. Son de este tipo de acción: prometer, garantizar, jurar ("juro que iré'), advertir, amenazar. La acción comunicativa se cumple en el presente, en el acto: 'te prometo'; el referente, en cambio: puede referirse a un tiempo futuro '..., que te voy a escribir'. La acción de prometer es solo eso que es: una acción ni verdadera ni falsa; su referente, 'lo prometido', será verdadero o falso cumplido el plazo de la promesa.

c) L actúa para que L', solo por el hecho de escuchar esta acción comunicativa, responda actuando de un modo correlativo y conmensurable a la acción de L (v. gr. Mandar-obedecer; pedir-dar; prohibir-obedecer, poner en disyuntiva-tomar uno de los disyuntos, etc.).

d) En virtud de la acción comunicativa L confiere a L' un atributo, una condición social. O impone un castigo, etc. (declarar reo, maldecir, bendecir, nombrar en un cargo, pedir la renuncia...). El logro o el malogro de este último tipo de acción no solo dependen del querer de quien las dice, sino de un poder reconocido por los otros para hacerlo.

e) Por su parte, L' responde de modo supuestamente correlativo y conmensurable a L, negando, negándose, protestando, contrargumentando, etc.

Ahora bien, referente y acción comunicativa son funciones, por llamarlas de alguna manera, inseparables pero distintas, de una misma estructura.

Es de una evidencia apodíctica que el referente -lo mostrado- no puede faltar en ninguna acción comunicativa, pero tampoco puede faltar la acción misma de mostrar, ahora, en el momento de la comunicación.

Que el referente no pueda faltar es algo comprensible de suyo: comunicar es comunicar algo; una acción necesariamente transitiva. Esto es: la acción no puede referirse a sí misma so pena de caer, como Epiménides, en la paradoja y en el sin sentido.

Pero en ninguna acción comunicativa, en cuanto acción, puede faltar un sujeto agente que la realice y, en cuanto comunicativa, un sujeto que responda a esa acción.

Es habitual, sin embargo, que lo que se muestra deje en penumbras a aquel que lo muestra: cuando abordamos un tema teórico, científico, filosófico, cuando nos referimos a las cosas que pasan; en general, podemos, sin más, prescindir del sujeto que está emitiendo una noticia, una opinión, un enunciado científico. La acción misma de transmitir, de hacer circular, de enseñar las cosas que importan, queda rezagada frente a la objetividad y a la importancia de lo que se dice como verdadero.

No obstante, es evidente que solo por el hecho de mostrarlo, el hecho vuelve a la actualidad del mundo, al presente. El presente verbal, ligado a la acción comunicativa, 
marca $^{13}$ la integración de todos los sujetos de una comunidad lingüística en la línea de un tiempo común. Tiempo común, mundo común, son favores que nos concede el habla.

\section{Referencias bibliográficas}

Alighieri, Dante (1949), La Divina Commedia, Paradiso. Milano: Biblioteca Universale Rizzoli. Versión en español: La divina comedia, traducción en verso ajustada al original con nuevos comentarios por Bartolomé Mitre, Buenos Aires: Jacobo Peuser, 1894.

Aristóteles (1986), Etica Nicomachea. Texto griego al frente, introducción, traducción y comentario de M. Zanetta. Milano: Biblioteca Universale Rizzoli. Versión en español: Ética a Nicómaco, edición bilingüe y traducción de María Araujo y Julián Marías, 6. ${ }^{a}$ edición. Madrid: Centro de Estudios Constitucionales, 1994.

(2000), Dell'Anima en: Opere, Vol. 4: Della generazione e della corruzione-Dell' Anima-Piccoli trattati di storia naturale. Texto griego al frente, introducción, traducción y comentario de A. Russo y R. Laurenti, Milano: Biblioteca Universale Laterza. Versión en español: Acerca del alma, introducción, traducción y notas de Tomaìs Calvo Martínez. Madrid: Editorial Gredos, 1978.

Fuentes, Juan José (2007), “El problema de la voluntad en H. Arendt: ¿un debate kantiano?", Daimon. 41: 77-90.

Heidegger, Martin (1997), Ser y Tiempo. Traducción al español de Jorge Eduardo Rivera. Santiago: Editorial Universitaria.

Wittgenstein, Ludwig (1987), Tractatus logico-philosophicus. Traduzione di Amedeo G. Conte. Torino: Einaudi.

13 Con el 'yo' comunicativo (no cartesiano) ocurre algo análogo a lo que ocurre con el instante (nyn) aristotélico. El instante es, él mismo, móvil, que va 'cortando' su paso por las diversas 'estaciones' que va haciendo, siempre en relación con otros móviles. 